PROCEEDINGS OF THE

AMERICAN MATHEMATICAL SOCIETY

Volume 135, Number 12, December 2007, Pages 3875-3885

S 0002-9939(07)08896-X

Article electronically published on August 29, 2007

\title{
ON THE INDEX AND SPECTRUM OF DIFFERENTIAL OPERATORS ON $\mathbb{R}^{N}$
}

\author{
PATRICK J. RABIER
}

(Communicated by David S. Tartakoff)

\begin{abstract}
If $P(x, \partial)$ is an $r \times r$ system of differential operators on $\mathbb{R}^{N}$ having continuous coefficients with vanishing oscillation at infinity, the Cordes-Illner theory ensures that $P(x, \partial)$ is Fredholm from $\left(W^{m, p}\right)^{r}$ to $\left(L^{p}\right)^{r}$ for all or no value $p \in(1, \infty)$. We prove that both the index (when defined) and the spectrum of $P(x, \partial)$ are independent of $p$.
\end{abstract}

\section{INTRODUCTION}

Let $P(x, \partial):=\sum_{|\alpha| \leq m} a_{\alpha}(x) \partial^{\alpha}$ be a differential operator on $\mathbb{R}^{N}$ with coefficients $a_{\alpha} \in \mathfrak{O}_{0}\left(\mathbb{R}^{N}\right)$, the space of bounded continuous complex-valued functions having vanishing oscillation at infinity (see Section 2). The Cordes-Illner theory, announced by Cordes in [2] but completed by Illner [12, gives a simple necessary and sufficient condition for $P(x, \partial)$ to be a Fredholm operator from the Sobolev space $W^{m, p}:=W^{m, p}\left(\mathbb{R}^{N}\right)$ to $L^{p}:=L^{p}\left(\mathbb{R}^{N}\right)$ for some $p \in(1, \infty)$. This condition can be generalized to systems $P(x, \partial):=\sum_{|\alpha| \leq m} A_{\alpha}(x) \partial^{\alpha}$ with $r \times r$ coefficient matrices $A_{\alpha}$ and it is independent of $p$ in all cases. Thus, the Fredholm property is $p$-independent, which suggests, but does not prove, that the index is $p$-independent as well. In that regard, it should be pointed out that the method of Cordes and Illner is based on the characterization of Fredholm operators by their invertibility modulo compact operators, which annihilates information about the index.

Unfortunately, Illner's exposition is not self-contained and many other sources must be checked for background material and several proofs. This, plus the heavy Banach algebra flavor, may explain why the theory has remained unknown to most potential users. In fact, twenty years after its publication, it was partially rediscovered by Fan and Wong [6] in 1997 by pseudodifferential operator methods which do not clarify the properties of the index.

For boundary value problems on bounded domains, the $p$-independence of the index was first observed by Geymonat 8 ] in 1965, and it continues to be investigated in ever greater generality (Kozhevnikov [13]). However, for problems in the whole space (or other unbounded domains) it has remained a mostly unanswered question, in spite of various related classical results.

Received by the editors January 14, 2006 and, in revised form, August 27, 2006.

2000 Mathematics Subject Classification. Primary 47A53, 47F05, 35J45.

Key words and phrases. Fredholm operator, index, differential operator, system, spectrum.

(C)2007 American Mathematical Society Reverts to public domain 28 years from publication 
For instance, Seeley [18] proved it for a class of elliptic singular integral operators on $L^{p}$ for which ellipticity is equivalent to Fredholmness, which is not the case with operators arising from PDEs on the whole space. Next, the Fedosov-Hörmander formula for the index (see (3.3)) does not depend upon $p$, but since it has only been proved when $p=2$, it does not say anything about $p$-independence. Lastly, in the weighted Sobolev spaces considered by McOwen [15] and others to discuss perturbations of the Laplace operator, neither the Fredholm property nor the index are $p$-independent. Among the positive results, Fredholm differential operators with constant coefficients are isomorphisms (an easy by-product of their commuting with translations), hence have index 0 irrespective of $p \in(1, \infty)$. Also, the $p$ independence of the index of Schrödinger operators with Kato-Rellich potentials is proved in [17.

In practice, the importance of $p \neq 2$ is crucial in many, if not most, nonlinear PDEs of order $m$ involving Fredholm operators, which can only be formulated in the $W^{m, p}$ setting with $p>2$. In such problems, the $p$-independence of the index of the linearization reduces its calculation to the case when $p=2$, in which more results and methods are available. This remark is expanded at the end of Section 3. Also, minimal smoothness of the coefficients is desirable since linearizations of nonlinear operators on $W^{m, p}$ do not have $\mathcal{C}^{\infty}$ coefficients.

In contrast to the index question, the investigation of the $p$-independence of the spectrum, a more specialized issue, has taken much momentum since the 1986 paper of Hempel and Voigt 9. Most of its subsequent generalizations are based on Gaussian estimates or functional calculus and often assume strong ellipticity and (at least) Hölder continuous coefficients. Relatively few discuss operators of arbitrary order and even fewer systems of arbitrary order; see the 1997 survey by Davies 4 for more information about hypotheses and methods. Once again, the $p$-independence of the spectrum is important in nonlinear problems (for the implicit function theorem or the existence of bifurcation points, among other issues) since the direct characterization of the spectrum is usually much simpler when $p=2$.

In this paper, we show that, under the sole assumption that the coefficients are in $\left(O_{0}\left(\mathbb{R}^{N}\right)\right)^{r \times r}$, the index of a system

$$
P(x, \partial):=\sum_{|\alpha| \leq m} A_{\alpha}(x) \partial^{\alpha}
$$

acting from $\left(W^{m, p}\right)^{r}$ to $\left(L^{p}\right)^{r}$ is, when defined, independent of $p \in(1, \infty)$ (Theorem 3.5). The proof is conceptually simple. However, the argument relies in part on a connection between the Fredholmness of the operator and the asymptotic behavior of the solutions observed only recently (16]).

As an application of Theorem 3.5 we also prove, without any additional assumption, that the spectrum of $P(x, \partial)$, viewed as an unbounded operator on $\left(L^{p}\right)^{r}$ with domain $\left(W^{m, p}\right)^{r}$, is also $p$-independent in the range $(1, \infty)$ (Corollary 4.5). Ellipticity is not needed explicitly but plays a major role insofar as failure of uniform ellipticity implies that the spectrum is the whole complex plane. Once again, in sharp contrast with many other treatments of the same problem, the proof uses only elementary concepts, even though our assumptions are more general in several respects. In fact, when the coefficients are smooth, spectral independence follows at once from the results of Section 3 and only the transition to continuous coefficients requires a careful examination. 


\section{The Cordes-Illner theorem}

In this section, we give a description of the main result of the Cordes-Illner theory. The space $\mathcal{C}_{b}\left(\mathbb{R}^{N}\right)$ of complex-valued bounded continuous functions on $\mathbb{R}^{N}$ is equipped with the uniform norm. Given $a \in \mathcal{C}_{b}\left(\mathbb{R}^{N}\right)$, the modulus of continuity $m c_{a}$ of $a$ is the nonnegative function on $\mathbb{R}^{N}$ defined by

$$
m c_{a}(x):=\sup \{|a(x)-a(y)|:|x-y| \leq 1\} .
$$

We define the space $\mathfrak{O}_{0}\left(\mathbb{R}^{N}\right)$ of bounded continuous functions on $\mathbb{R}^{N}$ with vanishing oscillation at infinity by

$$
\mathfrak{O}_{0}\left(\mathbb{R}^{N}\right):=\left\{a \in \mathcal{C}_{b}\left(\mathbb{R}^{N}\right): \lim _{|x| \rightarrow \infty} m c_{a}(x)=0\right\} .
$$

The main result of the Cordes-Illner theory reads as follows (in a specialized form sufficient for our purposes):

Theorem 2.1. Assume that $A_{\alpha} \in\left(\mathfrak{O}_{0}\left(\mathbb{R}^{N}\right)\right)^{r \times r},|\alpha| \leq m$. The operator $P(x, \partial):=$ $\sum_{|\alpha| \leq m} A_{\alpha}(x) \partial^{\alpha}$ is Fredholm from $\left(W^{m, p}\right)^{r}$ to $\left(L^{p}\right)^{r}$ for some $p \in(1, \infty)$ if and only if there are constants $c>0$ and $\rho>0$ such that

$$
\left|\operatorname{det}\left(\sum_{|\alpha| \leq m} A_{\alpha}(x) i^{|\alpha|} \xi^{\alpha}\right)\right| \geq c\left(1+|\xi|^{2}\right)^{\frac{m r}{2}}, \quad(x, \xi) \in \mathbb{R}^{N}:|x|+|\xi| \geq \rho .
$$

While not developed by Cordes (except when $p=2$, a nontypical case) or Illner, the theory for systems $(r \geq 2)$ is discussed by Sun [19. A proof of Theorem 2.1 for $p=2$ also appears in Hörmander [1], under some restrictions about the coefficients. When $p=2$ and $r=1$, an earlier proof was given by Taylor 20] with the help of the $C^{*}$-algebra apparatus, not available when $p \neq 2$.

By dividing both sides of (2.2) by $\left(1+|\xi|^{2}\right)^{\frac{m r}{2}}$ and letting $|\xi| \rightarrow \infty$, we obtain $\left|\operatorname{det}\left(\sum_{|\alpha|=m} A_{\alpha}(x) \eta^{\alpha}\right)\right| \geq c$ for every $(x, \eta) \in \mathbb{R}^{N} \times \mathbb{S}^{N-1}\left(\mathbb{S}^{N-1}\right.$ the unit sphere of $\mathbb{R}^{N}$ ). Thus, (2.2) entails the uniform Petrovsky ellipticity condition

$$
\left|\operatorname{det}\left(\sum_{|\alpha|=m} A_{\alpha}(x) \xi^{\alpha}\right)\right| \geq c|\xi|^{m r}, \quad \forall(x, \xi) \in \mathbb{R}^{N},
$$

but note that (2.2) also depends upon the lower-order terms. When $r=1$, (2.3) reduces to the usual uniform ellipticity condition.

\section{The $p$-INDEPENDENCE OF THE INDEX}

We shall now complement Theorem 2.1 by showing that not only the Fredholm property, but also the index of $P(x, \partial):=\sum_{|\alpha| \leq m} A_{\alpha}(x) \partial^{\alpha}$, is independent of $p \in(1, \infty)$. To distinguish $P(x, \partial)$ acting from $\left(W^{m, p}\right)^{r}$ to $\left(L^{p}\right)^{r}$ for various values of $p$, we shall use the notation

$$
P_{p}(x, \partial):=P(x, \partial) \in \mathcal{L}\left(\left(W^{m, p}\right)^{r},\left(L^{p}\right)^{r}\right) .
$$

Therefore, " $P_{p}(x, \partial)$ is Fredholm" means that $P(x, \partial)$ is Fredholm from $\left(W^{m, p}\right)^{r}$ to $\left(L^{p}\right)^{r}$. In what follows, we shall make repeated use of the space

$$
\mathfrak{O}_{0}^{\infty}\left(\mathbb{R}^{N}\right):=\left\{a \in \mathcal{C}^{\infty}\left(\mathbb{R}^{N}\right) \cap \mathcal{C}_{b}\left(\mathbb{R}^{N}\right): \lim _{|x| \rightarrow \infty} \partial^{\alpha} a(x)=0, \forall|\alpha| \geq 1\right\} .
$$


The first result we need is the denseness of $\mathfrak{O}_{0}^{\infty}\left(\mathbb{R}^{N}\right)$ in $\mathfrak{O}_{0}\left(\mathbb{R}^{N}\right)$ (see (2.1)), which is easily established by a mollification procedure (see [3, Lemma 2]). For future reference, we record it in

Lemma 3.1. The space $\mathfrak{O}_{0}^{\infty}\left(\mathbb{R}^{N}\right)$ is dense in $\mathfrak{O}_{0}\left(\mathbb{R}^{N}\right)$ for the uniform topology.

The next lemma is more or less folklore, but apparently hard to find in print. It states that the regularity of the solutions depends only upon the Fredholmness of the operator $P(x, \partial)$, with no need for any explicit ellipticity assumption. Of course, ellipticity is embodied by the equivalent condition (2.2) for Fredholmness, but it is noteworthy that (2.2) does not incorporate strong ellipticity.

Lemma 3.2. Let $P(x, \partial):=\sum_{|\alpha| \leq m} A_{\alpha}(x) \partial^{\alpha}$, where $A_{\alpha} \in\left(\mathfrak{O}_{0}^{\infty}\left(\mathbb{R}^{N}\right)\right)^{r \times r},|\alpha| \leq$ $m$. Assume that $P_{p}(x, \partial)$ is Fredholm for some $p \in(1, \infty)$. If $U \in\left(W^{m, p}\right)^{r}$ and $P(x, \partial) U \in\left(W^{k, p}\right)^{r}$ for some $k \geq 0$, then $U \in\left(W^{m+k, p}\right)^{r}$.

Proof. In this proof, the norm of $\left(W^{s, p}\right)^{r}$ is denoted by $\|\cdot\|_{s, p}\left(\left(L^{p}\right)^{r}\right.$ norm if $\left.s=0\right)$. Since $P_{p}(x, \partial)$ is Fredholm, there is a constant $C>0$ such that

$$
\|U\|_{m, p} \leq C\left(\|P(x, \partial) U\|_{0, p}+\|U\|_{0, p}\right),
$$

for every $U \in\left(W^{m, p}\right)^{r}$. Indeed, $\|P(x, \partial) U\|_{0, p}+\|U\|_{0, p}$ is clearly a norm on $\left(W^{m, p}\right)^{r}$, so that (3.2) follows from the open mapping theorem if we show that $\left(W^{m, p}\right)^{r}$ is complete for this norm. In turn, this amounts to proving that if $\left(U_{n}\right) \subset\left(W^{m, p}\right)^{r}$ and both $\left(P(x, \partial) U_{n}\right)$ and $\left(U_{n}\right)$ converge in $L^{p}$, then $\left(U_{n}\right)$ converges in $\left(W^{m, p}\right)^{r}$.

Let $E \in \mathcal{L}\left(\left(W^{m, p}\right)^{r}\right)$ denote a projection onto $\operatorname{ker} P_{p}(x, \partial)$. Since $P_{p}(x, \partial)$ is Fredholm, the convergence of $\left(P(x, \partial) U_{n}\right)$ in $\left(L^{p}\right)^{r}$ entails the convergence of $\left((I-E) U_{n}\right)$ in $\left(W^{m, p}\right)^{r}$ and hence in $\left(L^{p}\right)^{r}$. Since $\left(U_{n}\right)$ converges in $\left(L^{p}\right)^{r}$, it follows that $\left(E U_{n}\right)$ converges in $\left(L^{p}\right)^{r}$. But on the finite-dimensional space $\operatorname{ker} P_{p}(x, \partial) \subset$ $\left(W^{m, p}\right)^{r}$, convergence in $\left(L^{p}\right)^{r}$ is the same as convergence in $\left(W^{m, p}\right)^{r}$. Thus, both $\left((I-E) U_{n}\right)$ and $\left(E U_{n}\right)$ converge in $\left(W^{m, p}\right)^{r}$, so that $\left(U_{n}\right)$ converges in $\left(W^{m, p}\right)^{r}$. This proves (3.2).

The coefficients $A_{\alpha}$ are smooth and bounded, with bounded derivatives of all orders. Therefore, by the well-known method of differential quotients and induction, it follows from (3.2) that, if $P(x, \partial) U \in\left(W^{k, p}\right)^{r}$ for some $k \geq 0$, then $U \in\left(W^{m+k, p}\right)^{r}$.

Lemma 3.3 below relies on the fact that Fredholmness alone implies the exponential decay of the solutions of PDEs with exponentially decaying right-hand sides $([16])$.

Lemma 3.3. Let $P(x, \partial):=\sum_{|\alpha| \leq m} A_{\alpha}(x) \partial^{\alpha}$, where $A_{\alpha} \in\left(\mathfrak{O}_{0}^{\infty}\left(\mathbb{R}^{N}\right)\right)^{r \times r},|\alpha| \leq$ $m$. Assume that $P_{p}(x, \partial)$ is Fredholm for some $p \in(1, \infty)$. If $U \in\left(W^{m, p}\right)^{r}$ and $P(x, \partial) U=\Phi \in\left(\mathcal{C}_{0}^{\infty}\left(\mathbb{R}^{N}\right)\right)^{r}$, then $U \in\left(W^{m, q}\right)^{r}$ for all $q \in[1, \infty]$.

Proof. Since $\Phi \in\left(W^{k, p}\right)^{r}$ for every $k \geq 0$, it follows from Lemma 3.2 that $U \in$ $\bigcap_{k \geq 0}\left(W^{m+k, p}\right)^{r} \subset\left(W^{m, \infty}\right)^{r}$. Since also $U \in\left(W^{m, p}\right)^{r}$, this shows that $U \in$ $\left(W^{m, q}\right)^{r}$ for every $q \in[p, \infty]$.

Now, denote by $\rho$ a $\mathcal{C}^{\infty}$ function on $\mathbb{R}^{N}$ such that $\rho \geq 0$ and $\rho(x)=|x|$ for $|x| \geq 1$. Then, all the derivatives $\partial^{\beta} \rho$ with $|\beta| \geq 1$ are bounded and, since $\Phi$ has compact support, it is obvious that $e^{s \rho} \Phi \in\left(L^{p}\right)^{r}$ for every $s>0$. Since $P_{p}(x, D)$ is Fredholm and $p \in(1, \infty)$ (so that $\left(L^{p}\right)^{r}$ is reflexive), it follows that there are $t>0$ 
and $V \in\left(W^{m, p}\right)^{r}$ such that $U=e^{-t \rho} V$. (See [16, Section 3.1], where the case $r=1$ is discussed and where it is pointed out that all the arguments carry over trivially to the case $r \geq 1$.) A straightforward verification based on Leibnitz' formula reveals that $e^{-t \rho}$ and all its derivatives have exponential decay as $|x| \rightarrow \infty$. In particular, $e^{-t \rho} \in W^{m, p^{\prime}}, p^{\prime}:=\frac{p}{p-1}$, so that $U=e^{-t \rho} V \in\left(W^{m, 1}\right)^{r}$. Since also $U \in\left(W^{m, p}\right)^{r}$, this shows that $U \in\left(W^{m, q}\right)^{r}$ for every $q \in[1, p]$. Thus, altogether, $U \in\left(W^{m, q}\right)^{r}$ for every $q \in[1, \infty]$.

The next lemma shows that an upper estimate for the codimension of a closed subspace $Z$ of a Banach space $Y$ can be obtained from considerations involving only a dense subspace of $Y$.

Lemma 3.4. Let $Y$ be a Banach space and let $Z \subset Y$ be a closed subspace. Suppose that $D \subset Y$ is a dense subspace and that there are $d_{1}, \ldots, d_{k} \in D$ with the following property: For every $w \in D$, there are scalars $\mu_{1}, \ldots, \mu_{k}$ such that $w-\sum_{i=1}^{k} \mu_{i} d_{i} \in Z$. Then, $\operatorname{codim} Z \leq k$.

Proof. Set $D_{0}:=\operatorname{span}\left\{d_{1}, \ldots, d_{k}\right\} \subset D$. If $D_{0} \subset Z$, then $D \subset Z$ and so $Z=Y$ since $D$ is dense and $Z$ is closed, and the result is obvious. From now on, we assume that $D_{0} \nsubseteq Z$. If $\left\{d_{1}^{\prime}, \ldots, d_{k^{\prime}}^{\prime}\right\}$ is a basis of any complement of $D_{0} \cap Z$ in $D_{0}$, then $k^{\prime} \leq k$ and $d_{1}^{\prime}, \ldots, d_{k^{\prime}}^{\prime}$ have the same properties as $d_{1}, \ldots, d_{k}$, namely, $d_{1}^{\prime}, \ldots, d_{k^{\prime}}^{\prime} \in D$ and, given $w \in D$, there are $\mu_{1}^{\prime}, \ldots, \mu_{k^{\prime}}^{\prime} \in \mathbb{C}$ such that $w-\sum_{i=1}^{k^{\prime}} \mu_{i}^{\prime} d_{i}^{\prime} \in Z$. Thus, if the lemma is true with $D_{0}$ replaced by $\operatorname{span}\left\{d_{1}^{\prime}, \ldots, d_{k^{\prime}}^{\prime}\right\}$, it is also true as stated. It follows that we may and shall assume that $d_{1}, \ldots, d_{k}$ are linearly independent and that $D_{0} \cap Z=\{0\}$.

By contradiction, assume that $\operatorname{codim} Z>k$. Since $\operatorname{dim} D_{0}=k$ and $D_{0} \cap Z=\{0\}$, there is $y \in Y \backslash D_{0}$ such that $\left(D_{0} \oplus \mathbb{C} y\right) \cap Z=\{0\}$. (The cosets $d_{1}+Z, \ldots, d_{k}+Z$ are linearly independent in $Y / Z$ and $\operatorname{dim} Y / Z>k$.) Since $D$ is dense in $Y$, the vector $y$ can be approximated by elements $w \in D$. If $w$ is close enough to $y$, then $w \notin D_{0}$. We claim that, in addition, $\left(D_{0} \oplus \mathbb{C} w\right) \cap Z=\{0\}$ (if $w$ is close enough to $y$ ).

To prove the claim, we also argue by contradiction: Otherwise, there is a sequence $\left(w_{n}\right) \subset D$ with $w_{n} \rightarrow y$ such that $\left(D_{0} \oplus \mathbb{C} w_{n}\right) \cap Z$ contains a sequence of nonzero vectors, say $v_{n}+\theta_{n} w_{n}$ with $v_{n} \in D_{0}$ and $\theta_{n} \in \mathbb{C}$. After rescaling, we may assume ||$v_{n}||+\left|\theta_{n}\right|=1$. If so, $\left(v_{n}\right)$ and $\left(\theta_{n}\right)$ are bounded and, since $D_{0}$ is finite dimensional, we may also assume that $v_{n} \rightarrow v \in D_{0}$ and $\theta_{n} \rightarrow \theta \in \mathbb{C}$. Then, $\|v\|+|\theta|=1$ and $v+\theta y \in Z$ by the closedness of $Z$. Since $\left(D_{0} \oplus \mathbb{C} y\right) \cap Z=\{0\}$, we infer that $v+\theta y=0$, which requires $\theta=0$ and $v=0$ since $y \notin D_{0}$. This contradicts $\|v\|+|\theta|=1$.

The above shows that, assuming codim $Z>k$, there is $w \in D \backslash D_{0}$ such that $\left(D_{0} \oplus \mathbb{C} w\right) \cap Z=\{0\}$. Now, since $w \in D$, the hypotheses of the lemma ensure that there are scalars $\mu_{1}, \ldots, \mu_{k}$ such that $w-\sum_{i=1}^{k} \mu_{i} d_{i} \in Z$. Since $w-\sum_{i=1}^{k} \mu_{i} d_{i} \in$ $D_{0} \oplus \mathbb{C} w$, it follows that $w-\sum_{i=1}^{k} \mu_{i} d_{i}=0$ and hence that $w \in D_{0}$, which is a contradiction.

Lemma 3.4 is trivially false if $Z$ is not closed in $Y$ (just let $Z=D$ and $k=0$ ).

Theorem 3.5. Let $P(x, \partial):=\sum_{|\alpha| \leq m} A_{\alpha}(x) \partial^{\alpha}$, where $A_{\alpha} \in\left(\mathfrak{O}_{0}\left(\mathbb{R}^{N}\right)\right)^{r \times r},|\alpha| \leq$ $m$. Assume that $P_{p}(x, \partial)$ is Fredholm for some $p \in(1, \infty)$. Then $P_{q}(x, \partial)$ is Fredholm for every $q \in(1, \infty)$ and index $P_{q}(x, \partial)=$ index $P_{p}(x, \partial)$. 
Proof. That $P_{q}(x, \partial)$ is Fredholm for every $q \in(1, \infty)$ follows from Theorem 2.1] With $q$ being now fixed, we prove that its index equals index $P_{p}(x, \partial)$. With no loss of generality, we may assume that $A_{\alpha} \in\left(\mathfrak{O}_{0}^{\infty}\left(\mathbb{R}^{N}\right)\right)^{r \times r},|\alpha| \leq m$. Indeed, recall that $\mathfrak{O}_{0}^{\infty}\left(\mathbb{R}^{N}\right)$ is dense in $\mathfrak{O}_{0}\left(\mathbb{R}^{N}\right)$ (Lemma 3.1) and note that a small perturbation of the coefficients $A_{\alpha}$ (in the uniform norm) induces a correspondingly small perturbation of both $P_{p}(x, \partial)$ in $\mathcal{L}\left(\left(W^{m, p}\right)^{r},\left(L^{p}\right)^{r}\right)$ and of $P_{q}(x, \partial)$ in $\mathcal{L}\left(\left(W^{m, q}\right)^{r},\left(L^{q}\right)^{r}\right)$, which therefore does not affect the Fredholm property or the index of $P_{p}(x, \partial)$ and $P_{q}(x, \partial)$.

With $\Phi=0$ in Lemma 3.3, we obtain at once that $\operatorname{ker} P_{p}(x, \partial)=\operatorname{ker} P_{q}(x, \partial)$. Therefore, to complete the proof, it only remains to show that codim rge $P_{p}(x, \partial)=$ codim rge $P_{q}(x, \partial)$. Set $k:=$ codim rge $P_{p}(x, \partial)$ and let $\Phi_{1}, \ldots, \Phi_{k}$ span a complement of rge $P_{p}(x, \partial)$ in $\left(L^{p}\right)^{r}$. By the denseness of $\left(\mathcal{C}_{0}^{\infty}\left(\mathbb{R}^{N}\right)\right)^{r}$ in $\left(L^{p}\right)^{r}$ and the closedness of rge $P(x, \partial)$, we may and shall assume that $\Phi_{i} \in\left(\mathcal{C}_{0}^{\infty}\left(\mathbb{R}^{N}\right)\right)^{r}, 1 \leq i \leq k$. Now, we use Lemma 3.4 with $X=\left(W^{m, q}\right)^{r}, Y=\left(L^{q}\right)^{r}, Z=\operatorname{rge} P_{q}(x, \partial)$ and $D=\left(\mathcal{C}_{0}^{\infty}\left(\mathbb{R}^{N}\right)\right)^{r}$. To ascertain that codim rge $P_{q}(x, \partial) \leq k$, the only thing to check is that, given $\Phi \in\left(\mathcal{C}_{0}^{\infty}\left(\mathbb{R}^{N}\right)\right)^{r}$, there are $\mu_{1}, \ldots, \mu_{k} \in \mathbb{C}$ such that $\Phi-\sum_{i=1}^{k} \mu_{i} \Phi_{i} \in$ rge $P_{q}(x, \partial)$. Since $\operatorname{span}\left\{\Phi_{1}, \ldots, \Phi_{k}\right\}$ is a complement of $\operatorname{rge} P_{p}(x, \partial)$, scalars $\mu_{1}, \ldots, \mu_{k}$ $\in \mathbb{C}$ can be found such that $\Phi-\sum_{i=1}^{k} \mu_{i} \Phi_{i} \in \operatorname{rge} P_{p}(x, \partial)$. Let $U \in\left(W^{m, p}\right)^{r}$ be such that $P(x, \partial) U=\Phi-\sum_{i=1}^{k} \mu_{i} \Phi_{i}$. Since the right-hand side is in $\left(\mathcal{C}_{0}^{\infty}\left(\mathbb{R}^{N}\right)\right)^{r}$, Lemma 3.3 shows that $U \in\left(W^{m, q}\right)^{r}$, so that indeed $\Phi-\sum_{i=1}^{k} \mu_{i} \Phi_{i} \in \operatorname{rge} P_{q}(x, \partial)$.

The above shows that codim rge $P_{p}(x, \partial) \geq \operatorname{codim} \operatorname{rge} P_{q}(x, \partial)$. Hence, equality holds since $p$ and $q$ are arbitrary.

Remark 3.1. If the coefficients of $P(x, \partial)$ are real, Theorem 3.5 remains true in the real case. This follows from the fact that the index of a Fredholm operator between real Banach spaces $X$ and $Y$ equals its index (over $\mathbb{C}$ ) as an operator between the complexifications of $X$ and $Y$.

For instance, Theorem 3.5 implies that if $P_{2}(x, \partial)$ is Fredholm and selfadjoint as an unbounded operator on $\left(L^{2}\right)^{r}$ with domain $\left(W^{m, 2}\right)^{r}$, then $P_{p}(x, \partial)$ has index 0 for $p \in(1, \infty)$.

On the other hand, under suitable additional assumptions about the coefficients $A_{\alpha}$ (see below), the index of $P_{p}(x, \partial)$ is given by

$$
\text { index } P_{p}(x, \partial)=-\left(\frac{i}{2 \pi}\right)^{N} \frac{(N-1) !}{(2 N-1) !} \int_{\partial B} \operatorname{Tr}\left(\left(\sigma^{-1} d \sigma\right)^{\wedge 2 N-1}\right),
$$

where $\sigma(x, \xi):=\sum_{|\alpha| \leq m} A_{\alpha}(x) i^{|\alpha|} \xi^{\alpha}$ is the symbol of $P(x, \partial), B$ is any open ball in $\mathbb{R}^{N} \times \mathbb{R}^{N}$ such that $\sigma(x, \xi)$ is invertible on the exterior of $B$ (recall condition (2.2), equivalent to the Fredholmness of $P_{p}(x, \partial)$ ), $\mathrm{Tr}$ is the (matrix) trace and $\left(\sigma^{-1} d \sigma\right)^{\wedge 2 N-1}:=\left(\sigma^{-1} d \sigma\right) \wedge \cdots \wedge\left(\sigma^{-1} d \sigma\right)(2 N-1$ factors $)$.

As noted by Bott and Seeley [1], when $p=2$, formula (3.3) follows from Hörmander [11, Theorem 7.3] and extends a prior result of Fedosov [7 for a smaller class of symbols. Theorem 3.5 implies its validity for all $p \in(1, \infty)$. The direct proof of (3.3) involves special features of $L^{2}$ and cannot be repeated when $p \neq 2$. Also, (3.3) has only been proved for smooth $\left(\mathcal{C}^{\infty}\right)$ coefficients $A_{\alpha}$ with derivatives satisfying

$$
\left|\partial^{\beta} A_{\alpha}(x)\right|=O\left(|x|^{-|\beta|}\right) \text { as }|x| \rightarrow \infty .
$$

It is a conjecture of Bott and Seeley that formula (3.3) is still valid when $A_{\alpha} \in$ $\left(\mathfrak{O}_{0}^{\infty}\left(\mathbb{R}^{N}\right)\right)^{r \times r}$, but we are not aware that the issue has been settled. 
By the stability of the index under small perturbations, (3.3) provides a way to calculate the index of $P_{p}(x, \partial)$ when the coefficients $A_{\alpha}$ are merely continuous but can be uniformly approximated by smooth coefficients satisfying (3.4). For instance, when $A_{\alpha}(x)$ has a constant limit $A_{\alpha}^{\infty}$ when $|x| \rightarrow \infty$.

When $r<N$, the right-hand side of (3.3) vanishes. This is implicit from parts of the discussion in 1. The argument may be briefly summarized as follows. For $k \in \mathbb{N}$, define the differential forms on $G L\left(\mathbb{C}^{r}\right)$ by

$$
\omega_{k}:=\operatorname{Tr}\left(\left(Z^{-1} d Z\right)^{\wedge k}\right),
$$

where $Z \in G L\left(\mathbb{C}^{r}\right)$. The forms $\omega_{k}$ are closed, and it is known that the de Rham cohomology of $G L\left(\mathbb{C}^{r}\right)$ is an exterior algebra with generators $\omega_{1}, \ldots, \omega_{2 r-1}$. For its most part, this result (quoted in 1] with no reference) follows from the fact that $G L\left(\mathbb{C}^{r}\right)$ has the same cohomology as the unitary group $\mathbb{U}(r)$ and from corresponding results for $\mathbb{U}(r)$. For the latter, see for instance Dieudonné [5, p. 352].

Accordingly, if $r<N$, then modulo exact forms, $\omega_{2 N-1}$ is a linear combination of terms $\omega_{k_{1}} \wedge \cdots \wedge \omega_{k_{\ell}}$ with $0<k_{j}<2 N-1$ and $k_{1}+\cdots+k_{\ell}=2 N-1$. Therefore, since the form $\operatorname{Tr}\left(\left(\sigma^{-1} d \sigma\right)^{\wedge 2 N-1}\right)$ is the pull-back $\sigma^{*} \omega_{2 N-1}$ of $\omega_{2 N-1}$ to $\partial B$, it is a linear combination of the forms $\sigma^{*} \omega_{k_{1}} \wedge \cdots \wedge \sigma^{*} \omega_{k_{\ell}}$ (modulo exact forms). Each form $\sigma^{*} \omega_{k_{j}}$ on $\partial B$ is closed and of order $k_{j} \in\{1, \ldots, 2 N-2\}$ and hence exact since $\partial B$ is a $(2 N-1)$-dimensional sphere. It follows that $\sigma^{*} \omega_{k_{1}} \wedge \cdots \wedge \sigma^{*} \omega_{k_{\ell}}$ is exact. Thus, $\operatorname{Tr}\left(\left(\sigma^{-1} d \sigma\right)^{\wedge 2 N-1}\right)=\sigma^{*} \omega_{2 N-1}$ is exact, so that its integral on $\partial B$ vanishes.

In particular, the above shows that, assuming (2.2), $P_{p}(x, \partial)$ has index 0 for all $p \in(1, \infty)$ when $r<N$ and the coefficients $A_{\alpha}$ are continuous with $\lim _{|x| \rightarrow \infty} A_{\alpha}(x)$ $=A_{\alpha}^{\infty}$. If strong ellipticity is assumed, an elementary proof of this result can be given, without the restriction $r<N$ : By a straightforward homotopy, the problem is reduced to the case when the leading coefficients are constant, so that $P_{p}(x, \partial)$ is a compact perturbation of a Fredholm operator with constant coefficients and therefore of index 0 .

\section{The $p$-IndePEndenCE OF THE SPECTRUM}

We now turn to the $p$-independence of the spectrum of

$$
P(x, \partial):=\sum_{|\alpha| \leq m} A_{\alpha}(x) \partial^{\alpha},
$$

viewed as an unbounded operator on $\left(L^{p}\right)^{r}$ with domain $\left(W^{m, p}\right)^{r}$. Upon replacing $P(x, \partial)-\lambda, \lambda \in \mathbb{C}$, by $P(x, \partial)$, it suffices to show that $P_{p}(x, \partial)$ and $P_{q}(x, \partial)$ are simultaneously invertible when $p, q \in(1, \infty)$. This is readily seen when $A_{\alpha} \in$ $\left(\mathfrak{O}_{0}^{\infty}\left(\mathbb{R}^{N}\right)\right)^{r \times r},|\alpha| \leq m$, for $P_{q}(x, \partial)$ is Fredholm of index 0 whenever $P_{p}(x, \partial)$ is invertible by Theorem 3.5, while $\operatorname{ker} P_{q}(x, \partial)=\operatorname{ker} P_{p}(x, \partial)(=\{0\})$ by Lemma 3.3. It is more demanding to resolve the case of coefficients $A_{\alpha} \in\left(\mathfrak{O}_{0}\left(\mathbb{R}^{N}\right)\right)^{r \times r}$, when the general $p$-independence of ker $P_{p}(x, \partial)$ need not be true. The argument for the proof will be developed in a string of lemmas. The first one is a straightforward variant of the foregoing remarks, still true when the coefficients are not smooth.

Lemma 4.1. Let $P(x, \partial):=\sum_{|\alpha| \leq m} A_{\alpha} \partial^{\alpha}$, where $A_{\alpha} \in\left(\mathfrak{O}_{0}\left(\mathbb{R}^{N}\right)\right)^{r \times r},|\alpha| \leq m$. Given $B_{\alpha} \in\left(\mathfrak{O}_{0}^{\infty}\left(\mathbb{R}^{N}\right)\right)^{r \times r},|\alpha| \leq m$, set $Q(x, \partial):=\sum_{|\alpha| \leq m} B_{\alpha} \partial^{\alpha}$. If $P_{p}(x, \partial)$ is invertible for some $p \in(1, \infty)$, there is $R>0$ such that if $\max _{|\alpha| \leq m}\left\|B_{\alpha}-A_{\alpha}\right\|_{\infty}<$ $R$, then $Q_{q}(x, \partial)$ is invertible for every $q \in(1, \infty)$. 
Proof. Since $P_{p}(x, \partial)$ is invertible, $Q_{p}(x, \partial)$ is also invertible when

$$
\left\|Q_{p}(x, \partial)-P_{p}(x, \partial)\right\|_{\mathcal{L}\left(\left(W^{m, p}\right)^{r},\left(L^{p}\right)^{r}\right)}
$$

is small enough and hence when $\max _{|\alpha| \leq m}\left\|B_{\alpha}-A_{\alpha}\right\|_{\infty}<R$ with $R>0$ small enough. By Theorem 3.5. $Q_{q}(x, \partial)$ is Fredholm of index 0 for every $q \in(1, \infty)$. In addition, since the coefficients of $Q(x, \partial)$ are in $\left(\mathfrak{O}_{0}^{\infty}\left(\mathbb{R}^{N}\right)\right)^{r \times r}$, Lemma 3.3 yields $\operatorname{ker} Q_{q}(x, \partial)=\operatorname{ker} Q_{p}(x, \partial)=\{0\}$. Thus, $Q_{q}(x, \partial)$ is invertible.

To prove that $P_{q}(x, \partial)$ is invertible if $P_{p}(x, \partial)$ is invertible when the coefficients are only continuous, we shall show that, otherwise, there is an operator $P^{\prime}(x, \partial):=$ $\sum_{|\alpha| \leq m} A_{\alpha}^{\prime} \partial^{\alpha}$ with $A_{\alpha}^{\prime} \in\left(\mathfrak{O}_{0}^{\infty}\left(\mathbb{R}^{N}\right)\right)^{r \times r},|\alpha| \leq m$, such that $\max _{|\alpha| \leq m}\left\|A_{\alpha}^{\prime}-A_{\alpha}\right\|_{\infty}$ is arbitrarily small and that $P_{q}^{\prime}(x, \partial)$ is not invertible. This contradicts Lemma 4.1. Once again, some of the main ingredients needed to establish the existence of $P^{\prime}(x, \partial)$ are more easily described in an abstract setting.

Lemma 4.2. Let $X$ and $Y$ be complex Banach spaces and let $T \in \mathcal{L}(X, Y)$ be Fredholm of index 0 . If $T$ is not invertible, there is an open ball $B(T, \rho) \subset \mathcal{L}(X, Y)$ and a complex-analytic mapping $G: B(T, \rho) \rightarrow \mathbb{C}$ such that $S \in B(T, \rho)$ is invertible if and only if $G(S) \neq 0$.

Proof. Let $X_{1}:=\operatorname{ker} T$ and $Y_{2}:=\operatorname{rge} T$ and let $X_{2}$ and $Y_{1}$ be closed (direct) complements of $X_{1}$ and $Y_{2}$, respectively (so that $\operatorname{dim} Y_{1}=\operatorname{dim} X_{1}<\infty$ ). For $i=$ 1,2 , call $E_{i}$ the projection onto $Y_{i}$ corresponding to the direct sum decomposition $Y=Y_{1} \oplus Y_{2}$. Then, every $S \in \mathcal{L}(X, Y)$ has the block decomposition

$$
S=\left(\begin{array}{ll}
S_{11} & S_{12} \\
S_{21} & S_{22}
\end{array}\right)
$$

where $S_{i j}:=E_{i} S_{\mid X_{j}}$. When $S=T$, then $T_{11}=0, T_{12}=0, T_{21}=0$ and $T_{22}$ is invertible. Since $S_{22}$ depends continuously upon $S$, there is an open ball $B(T, \rho) \subset$ $\mathcal{L}(X, Y)$ such that $S_{22}$ is invertible for every $S \in B(T, \rho)$. If so, the invertibility of $S$ amounts to the invertibility of $S_{11}-S_{12} S_{22}^{-1} S_{21} \in \mathcal{L}\left(X_{1}, Y_{1}\right)$ and hence to $G(S):=\operatorname{det}\left(S_{11}-S_{12} S_{22}^{-1} S_{21}\right) \neq 0$. Clearly, $G$ is complex-analytic on $B(T, \rho)$ (being a composite of such mappings).

The following comments may help clarify the purpose of Lemma 4.3 below. Suppose that $X \hookrightarrow Y$ and that $T \in \mathcal{L}(X, Y)$ is Fredholm of index 0 and not invertible. Then, 0 is an eigenvalue of $T$ viewed as an unbounded operator on $Y$ with domain $X$. If, in addition, 0 is an isolated eigenvalue of $T$, the spectrum of every $S \in \mathcal{L}(X, Y)$ close enough to $T$ contains a point $z$ arbitrarily close to 0 , i.e., $S-z I$ is not invertible. Thus, $S-z I$ provides a small and noninvertible perturbation of $T$ whenever $S$ is close to $T$. This property fails if 0 is not an isolated eigenvalue of $T$ : There may be operators $S$, arbitrarily close to $T$, such that $S-z I$ is always invertible for $|z|$ small. Lemma 4.3 shows that, given $H \in \mathcal{L}(X, Y)$, noninvertible small perturbations $S+z H$ of $T$ can still be found, for every $S$ close to $T$, provided that $T+H$ is invertible.

Lemma 4.3. Let $X$ and $Y$ be complex Banach spaces and let $T \in \mathcal{L}(X, Y)$ be Fredholm of index 0 and not invertible. There is an open ball $B(0, \rho) \subset \mathcal{L}(X, Y)$ with the following property: Given $H \in B(0, \rho)$ such that $T+H$ is invertible and $\varepsilon>0$, there is $\delta \in(0, \varepsilon]$ such that if $S \in B(T, \delta) \subset \mathcal{L}(X, Y)$, then $S+z H$ is not invertible for some $z \in \mathbb{C}$ with $|z|<\varepsilon$. 
Proof. Let $B(T, \rho)$ be given by Lemma 4.2 and let $H \in B(0, \rho)$ be such that $T+H$ is invertible. With $G: B(T, \rho) \rightarrow \mathbb{C}$ also from Lemma 4.2, the function $\gamma(z):=$ $G(T+z H) \in \mathbb{C}$ is holomorphic on some open neighborhood of the unit disk in $\mathbb{C}$ and $\gamma(1)=G(T+H) \neq 0=G(T)=\gamma(0)$. Thus, $\gamma$ is not constant, so that $z=0$ is an isolated zero of $\gamma$.

Let $\omega_{\varepsilon}$ denote the open disk in $\mathbb{C}$ with center 0 and radius $\varepsilon>0$. If $\varepsilon \in(0,1]$ is small enough, then $\gamma$ is holomorphic on $\omega_{\varepsilon}$, continuous on $\bar{\omega}_{\varepsilon}$ and $\gamma(z) \neq 0$ for every $z \in \partial \omega_{\varepsilon}$. This implies that the Brouwer degree $\operatorname{deg}\left(\gamma, \omega_{\varepsilon}, 0\right)$ is well defined and nonzero. Recall that for holomorphic functions, the Brouwer degree provides an exact count of the solutions (with multiplicity). Thus, $\operatorname{deg}\left(\gamma, \omega_{\varepsilon}, 0\right) \geq 1$ since $0 \in \omega_{\varepsilon}$ and $\gamma(0)=0$.

If $\delta>0$ is small enough, then $S+z H \in B(T, \rho)$ whenever $S \in B(T, \delta) \subset \mathcal{L}(X, Y)$ and $z \in \bar{\omega}_{\varepsilon}$. Furthermore, after shrinking $\delta$ if necessary, the continuity of $G$ shows that $G(S+z H) \neq 0$ when $z \in \partial \omega_{\varepsilon}$ and $S \in B(T, \delta)$ (otherwise, $\gamma$ would also vanish on $\left.\partial \omega_{\varepsilon}\right)$. Of course, it is not restrictive to assume that $\delta \leq \varepsilon$. Thus, given $S \in B(T, \delta)$, the function $h(t, z)=G(t S+(1-t) T+z H)$ is well defined and continuous on $[0,1] \times \bar{\omega}_{\varepsilon}$ and $h(t, z) \neq 0$ for $(t, z) \in[0,1] \times \partial \omega_{\varepsilon}$. Since $h(0, \cdot)=\gamma$ and $\operatorname{deg}\left(\gamma, \omega_{\varepsilon}, 0\right) \neq 0$, the homotopy invariance of the Brouwer degree shows that there is $z \in \omega_{\varepsilon}$ such that $h(1, z):=G(S+z H)=0$. By Lemma 4.2, $S+z H$ is not invertible. This proves the lemma when $\varepsilon \in(0,1]$ is small enough and hence when $\varepsilon>0$ is arbitrary.

Theorem 4.4. Let

$$
P(x, \partial):=\sum_{|\alpha| \leq m} A_{\alpha}(x) \partial^{\alpha},
$$

where $A_{\alpha} \in\left(\mathfrak{O}_{0}\left(\mathbb{R}^{N}\right)\right)^{r \times r},|\alpha| \leq m$. Assume that $P_{p}(x, \partial)$ is invertible for some $p \in(1, \infty)$. Then $P_{q}(x, \partial)$ is invertible for every $q \in(1, \infty)$.

Proof. By contradiction, suppose that $P_{q}(x, \partial)$ is not invertible for some $q \in(1, \infty)$. Let $R>0$ be given by Lemma 4.1 and, with $X:=\left(W^{m, q}\right)^{r}, Y:=\left(L^{q}\right)^{r}$ and $T:=P_{q}(x, \partial)$, let $\rho>0$ be given by Lemma 4.3. Recalling Lemma 3.1 (denseness of $\mathfrak{O}_{0}^{\infty}\left(\mathbb{R}^{N}\right)$ in $\left.\mathfrak{O}_{0}\left(\mathbb{R}^{N}\right)\right)$, choose $\widetilde{A}_{\alpha} \in\left(\mathfrak{O}_{0}^{\infty}\left(\mathbb{R}^{N}\right)\right)^{r \times r},|\alpha| \leq m$, such that $\max _{|\alpha| \leq m}\left\|\widetilde{A}_{\alpha}-A_{\alpha}\right\|_{\infty}<R$ and $\left\|\widetilde{P}_{q}(x, \partial)-P_{q}(x, \partial)\right\|_{\mathcal{L}\left(\left(W^{q}\right)^{r},\left(L^{q}\right)^{r}\right)}<\rho$, where

$$
\widetilde{P}(x, \partial):=\sum_{|\alpha| \leq m} \widetilde{A}_{\alpha}(x) \partial^{\alpha} .
$$

By Lemma 4.1, $\widetilde{P}_{q}(x, \partial)$ is invertible.

Now, write $\widetilde{P}_{q}(x, \partial)=P_{q}(x, \partial)+\left(\widetilde{P}_{q}(x, \partial)-P_{q}(x, \partial)\right)$. Since the invertibility of $\widetilde{P}_{q}(x, \partial)$ is not affected by small perturbations, we may approximate $\widetilde{P}_{q}(x, \partial)-$ $P_{q}(x, \partial)$ by $Q_{q}(x, \partial)$, where

$$
Q(x, \partial):=\sum_{|\alpha| \leq m} B_{\alpha}(x) \partial^{\alpha}
$$

has coefficients in $\left(\mathfrak{O}_{0}^{\infty}\left(\mathbb{R}^{N}\right)\right)^{r \times r}$, in such a way that $\left\|Q_{q}(x, \partial)\right\|_{\mathcal{L}\left(\left(W^{m, q}\right)^{r},\left(L^{q}\right)^{r}\right)}<\rho$ and that $P_{q}(x, \partial)+Q_{q}(x, \partial)$ is invertible.

Given $\varepsilon>0$, it follows from Lemma 4.3 that there is $\delta \in(0, \varepsilon]$ such that, for every $S(x, \partial):=\sum_{|\alpha| \leq m} C_{\alpha}(x) \partial^{\alpha}$ with $C_{\alpha} \in\left(\mathfrak{O}_{0}\left(\mathbb{R}^{N}\right)\right)^{r \times r},|\alpha| \leq m$, satisfying $\left\|S_{q}(x, \partial)-P_{q}(x, \partial)\right\|_{\mathcal{L}\left(\left(W^{m, q}\right)^{r},\left(L^{q}\right)^{r}\right)}<\delta$, there is $z \in \mathbb{C}$ with $|z|<\varepsilon$ such that $S_{q}(x, \partial)+z Q_{q}(x, \partial)$ is not invertible. 
By using once again the denseness of $\mathfrak{O}_{0}^{\infty}\left(\mathbb{R}^{N}\right)$ in $\mathfrak{O}_{0}\left(\mathbb{R}^{N}\right)$, the operator $S(x, \partial)$ above can be chosen with coefficients $C_{\alpha} \in\left(\mathfrak{O}_{0}^{\infty}\left(\mathbb{R}^{N}\right)\right)^{r \times r}$ arbitrarily close to $A_{\alpha}$. In particular, we may assume not only that $\left\|S_{q}(x, \partial)-P_{q}(x, \partial)\right\|_{\mathcal{L}\left(\left(W^{m, q}\right)^{r},(L q)^{r}\right)}<$ $\delta$, but also that $\max _{|\alpha| \leq m}\left\|C_{\alpha}-A_{\alpha}\right\|_{\infty}<\frac{R}{2}$. Therefore, the differential operator $S_{q}(x, \partial)+z Q_{q}(x, \partial)$ has coefficients $C_{\alpha}+z B_{\alpha} \in\left(\mathfrak{O}_{0}^{\infty}\left(\mathbb{R}^{N}\right)\right)^{r \times r}$ satisfying $\max _{|\alpha| \leq m}\left\|C_{\alpha}+z B_{\alpha}-A_{\alpha}\right\|_{\infty}<\frac{R}{2}+\varepsilon \max _{|\alpha| \leq m}\left\|B_{\alpha}\right\|_{\infty}$. As a result, if $\varepsilon$ is chosen so that $\varepsilon \max _{|\alpha| \leq m}\left\|B_{\alpha}\right\|_{\infty}<\frac{R}{2}$ in the first place, then $\max _{|\alpha| \leq m}\left\|C_{\alpha}+z B_{\alpha}-A_{\alpha}\right\|_{\infty}<$ $R$, and hence $S_{q}(x, \partial)+z Q_{q}(x, \partial)$ must be invertible by Lemma 4.1. This contradiction completes the proof.

The following corollary is obvious.

Corollary 4.5. Let $P(x, \partial):=\sum_{|\alpha| \leq m} A_{\alpha}(x) \partial^{\alpha}$, where $A_{\alpha} \in\left(\mathfrak{O}_{0}\left(\mathbb{R}^{N}\right)\right)^{r \times r},|\alpha| \leq$ $m$. If $P_{p}(x, \partial)$ is viewed as an unbounded operator on $\left(L^{p}\right)^{r}$ with domain $\left(W^{m, p}\right)^{r}$, the spectrum of $P_{p}(x, \partial)$ is independent of $p \in(1, \infty)$.

As noted in the Introduction, among the various results related to Corollary 4.5 in the literature, few are valid for systems of arbitrary order. Nevertheless, such results can be found in the works of Leopold and Schrohe 14] (when the coefficients are $\mathcal{C}^{\infty}$ ) and Hieber and Schrohe 10] (when the coefficients are Hölder continuous and the system is strongly elliptic). Both accommodate coefficients with nonvanishing oscillation at infinity.

No ellipticity or smoothness of the coefficients is needed in Corollary 4.5, but if the uniform Petrovsky ellipticity condition (2.3) fails to hold, then $P_{p}(x, \partial)-\lambda$ is not even Fredholm for any $\lambda \in \mathbb{C}$ by Theorem 2.1, so that the spectrum of $P_{p}(x, \partial)$ is all of $\mathbb{C}$ for every $p \in(1, \infty)$. While it is easy to speculate that the vanishing oscillation assumption could be removed without introducing extra assumptions, there seems to be little evidence to support such a conjecture at this time.

\section{REFERENCES}

[1] Bott, R. and Seeley, R., Some remarks on the paper of Callias: "Axial anomalies and index theorems on open spaces" [Comm. Math. Phys. 62 (1978) 213-234], Comm. Math. Phys. 62 (1978) 235-245. MR:507781 (80h:58045b)

[2] Cordes, H. O., Beispiele von Pseudo-Differentialoperator-Algebren, Applicable Anal. 2 (1972) 115-129. MR0402541(53:6360)

[3] Cordes, H. O., On compactness of commutators of multiplications and convolutions, and boundedness of pseudodifferential operators, J. Funct. Anal. 18 (1975) 115-131. MR0377599 $(51: 13770)$

[4] Davies, E. B., $L^{p}$ spectral theory of higher-order elliptic differential operators, Bull. London Math. Soc. 29 (1997) 513-546. MR1458713 (98d:35164)

[5] Dieudonné, J., Éléments d'analyse, Vol. 9, Gauthier-Villars, Paris, 1982. MR658305 (84a:57021)

[6] Fan, Q. and Wong, M. W., A characterization of Fredholm pseudo-differential operators, J. London Math. Soc. (2) 55 (1997) 139-145. MR1423291 (97j:47073)

[7] Fedosov, B. V., Analytical formulas for the index of elliptic operators, Trans. Mosc. Math. Soc. 30 (1974) 159-240. MR0420731 (54:8743)

[8] Geymonat, G., Sui problemi ai limiti per i sistemi lineari ellittici, Ann. Mat. Pura Appl. 69 (1965) 207-284. MR0196262 (33:4454)

[9] Hempel, R. and Voigt, J., The spectrum of a Schrödinger operator in $L^{p}\left(\mathbb{R}^{\nu}\right)$ is independent of p, Comm. Math. Phys. 104 (1986), 243-250. MR836002 (87h:35247)

[10] Hieber, M. and Schrohe, E., $L^{p}$ spectral independence of elliptic operators via commutator estimates, Positivity 3 (1999) 259-272. MR1708648 (2000e:35034) 
[11] Hörmander, L., The Weyl calculus of pseudodifferential operators, Comm. Pure Appl. Math. 32 (1979) 360-444. MR517939 (80j:47060)

[12] Illner, R., On algebras of pseudo differential operators in $L^{p}\left(\mathbb{R}^{n}\right)$, Comm. Partial Differ. Eq. 2 (1977) 359-393. MR0442758 (56:1137b)

[13] Kozhevnikov, A., Complete scale of isomorphisms for elliptic pseudodifferential boundaryvalue problems, J. London Math. Soc. 64 (2001) 409-422. MR.1853460 (2002j:35333)

[14] Leopold, H.-G. and Schrohe, E., Invariance of the $L_{p}$ spectrum for hypoelliptic operators, Proc. Amer. Math. Soc., 125 (1997) 3679-3687. MR1423315 (98b:35125)

[15] McOwen, R. C., The behavior of the Laplacian on weighted Sobolev spaces, Comm. Pure Appl. Math. 32 (1979) 783-795. MR539158 (81m:47069)

[16] Rabier, P. J., Fredholm operators, semigroups and the asymptotic and boundary behavior of solutions of PDEs, J. Differ. Eq. 193 (2003) 460-480. MR1998964 (2004f:47059) Corrigendum, J. Differ. Eq. 237 (2007) 257.

[17] Rabier, P. J. and Stuart, C. A., Fredholm properties of Schrödinger operators in $L^{p}\left(\mathbb{R}^{N}\right)$, Differ. Integral Eq. 13 (2000) 1429-1444. MR1787075 (2001m:47103)

[18] Seeley, R. T., The index of elliptic systems of singular integral operators, J. Math. Anal Appl., 7 (1963) 289-309. MR0159247(28:2464)

[19] Sun, S. H., A Banach algebra approach to the Fredholm theory of pseudodifferential operators, Sci. Sinica, Series A, 27 (1984) 337-344. MR763970(86b:47092)

[20] Taylor, M. E., Gelfand theory of pseudo differential operators and hypoelliptic operators, Trans. Amer. Math. Soc. 153 (1971), 495-510. MR0415430(54:3517)

Department of Mathematics, University of Pittsburgh, Pittsburgh, Pennsylvania 15260

E-mail address: rabier@imap.pitt.edu 